\title{
Optimizing the Transmission Range in an Underwater Acoustic Network
}

\author{
Arnau Porto and Milica Stojanovic \\ Massachusetts Institute of Technology \\ Cambridge, MA, USA \\ E-mail: porto@mit.edu, millitsa@mit.edu
}

\begin{abstract}
An extension of Distance-Aware Collision Avoidance Protocol (DACAP) [1] is proposed that permits its implementation in large networks where maximal connectivity is not available. The technique proposed increases the energy efficiency by optimizing the transmission power of the nodes. The optimal power is the minimal power that still guarantees connectivity between each node and the sink. Simulation results show that this transmission power also results in throughput maximization. For a network of nodes uniformly distributed within a rectangular grid, the optimal transmission range can be determined as a function of the node density. A closed form approximation for this dependence is obtained.
\end{abstract}

Index Terms-MAC, power control, optimal transmission range, underwater acoustic networks, energy efficiency, throughput.

\section{INTRODUCTION}

Underwater acoustic networks present several challenges for the design of medium access control (MAC) protocols. First, latency is very high because of the low speed of sound (nominally $1500 \mathrm{~m} / \mathrm{s}$ ). Additionally, attenuation depends both on the distance $l$ and the frequency of the signal $f$, as

$$
A(l, f)=l^{k} \cdot a(f)^{l}
$$

where $k$ is the spreading factor and $a(f)$ is the absorption coefficient, which increases with frequency. As a consequence, bandwidth is limited, and it decreases with transmission distance [2]. Finally, because the network lifetime depends on the batteries of the nodes, minimizing energy consumption is an important design goal.

In this paper we are focusing on random channel access, which is useful in scenarios with a large number of nodes, which transmit in a bursty manner at a relatively low duty cycle. Aloha is the predecessor and the basis of many contention oriented protocols [3]. However, its high collision rate requires too much power in underwater acoustic systems [1]. At the same time, because of the high latency, carrier sensing is not reliable. For this reason, MACA (Multiple Access with Collision Avoidance) [4] type of protocols have been considered for underwater networks. MACA is based on an exchange of RTS/CTS (request to send / clear to send) commands that secure the communication channel. This protocol has been used in an experimental underwater network [5]. Current research is focusing on MACA type protocols such as S-FAMA (SlottedFloor Acquisition Multiple Access) [6], PCAP (Propagationdelay-tolerant Collision-Avoidance Protocol) [7] and DACAP (Distance-Aware Collision Avoidance Protocol) [1]. Another approach considered for channel sharing in emergy-limited

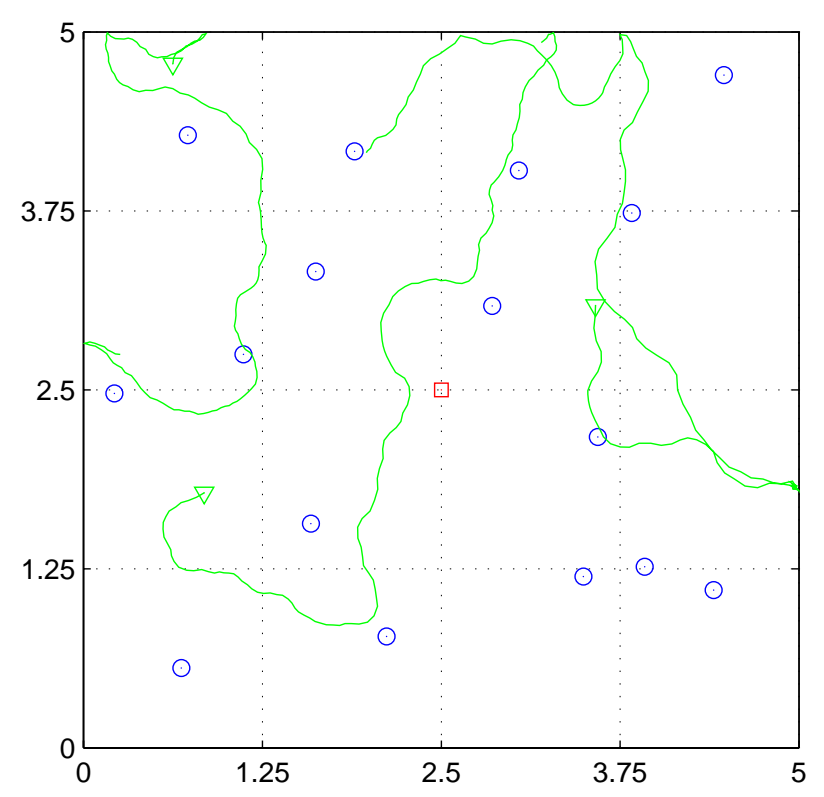

Fig. 1. Simulation scenario: $25 \mathrm{~km}^{2}, 16$ relays (circles), three AUVs (triangles with associated trajectories) and one sink (square in the middle).

underwater acoustic networks is that of sleeping schedules [8], [9].

DACAP offers good throughput performance, and, unlike S-FAMA, it is suitable for asynchronous nodes. DACAP was designed for applications with and without acknowledgments. However, it was only analyzed for maximally connected networks with fixed nodes. Maximal connectivity is achieved when any node can communicate with any other node via a single hop.

Our goal is to extend DACAP to situations without maximal connectivity, so as to make it scalable to networks with large coverage. In doing so, we aim to optimize the transmission power so as to minimize the overall power consumption. Optimization of the transmission range was considered in [10] for terrestrial radio networks; however, no similar analyses are available for underwater acoustic networks.

The paper is organized as follows. In Sec.II, DACAP is overviewed. In Sec.III, optimization of the transmission range is discussed and simulation results are presented. Sec.IV. establishes the relationship between transmission range and node density for a network with uniform node distribution. 


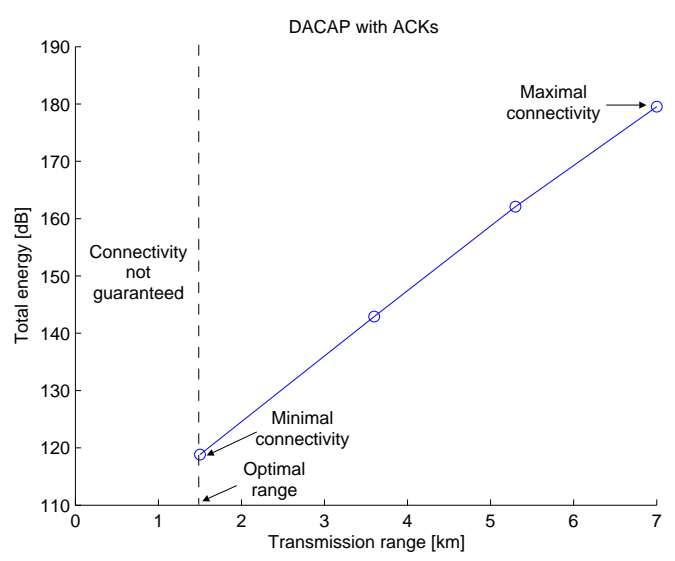

Fig. 2. Total energy consumption using the DACAP protocol with acknowledgments for different transmission ranges. Simulation time is 1000 seconds.

\section{OVERVIEW OF DACAP}

DACAP is a MAC protocol that has recently been proposed for underwater networks. This protocol implements a mechanism for avoiding packet collisions, thus maximizing the network throughput. In addition, it does not require the nodes to be synchronous. The DACAP protocol is based on the following steps:

- Upon receiving an RTS, a node sends CTS, and waits for a data packet. If during waiting, another RTS is overheard, the node sends a short warning to its partner.

- Upon receiving a CTS, a node waits for some time before transmitting the data packet. If during the waiting time another CTS is overheard, or if a warning arrives, the transmission is postponed by a random back-off time.

The waiting time is a function of the round-trip delay, which is proportional to the distance between the nodes and can be estimated after the handshake. DACAP capitalizes on the receiver's tolerance to interference when the transmitter and receivers are closer than the transmission range. The details of the protocol can be found in [1].

\section{ENERGY CONSUMPTION, THROUGHPUT, AND THE OPTIMAL TRANSMISSION RANGE}

We look at a scenario with a certain density of randomly distributed nodes, both mobile and fixed (see Fig.1). The nodes are assumed to transmit at the same power.

If the transmission power is large enough, then maximal connectivity is obtained, but the level of interference is high, causing a reduction in throughput. Reducing the transmission power thus has several implications. First, the total power consumption of the network is lower, since the power required to transmit a signal over multiple short hops is less than the power needed to transmit a signal over a single long hop. Secondly, interference levels are also lower, implying fewer collisions, and fewer postponements in transmission. However, the power cannot be reduced beyond the point where connectivity is lost. Hence, there is a trade-off in choosing the power level that still guarantees some degree of connectivity, but minimizes the overall power consumption and the interference levels. Note that as the power level is lowered from that needed to establish maximal connectivity, multi-hop routing becomes necessary.

We look at the shortest path routing and we ask the following question: Is there an optimal transmission power to be used by the nodes? We address this question from the viewpoint of minimizing the total power consumption and maximizing the total throughput.

The performance of the network is assessed by measuring the total energy consumption and the total throughput for different offered loads. The transmission power $P$ is related to the transmission range $R$ approximately as

$$
P=S N R \cdot A\left(f_{c}, R\right) \cdot N\left(f_{c}\right) \cdot B
$$

where $N\left(f_{c}\right)$ is the power spectral density of the noise, evaluated as in [2], $A\left(f_{c}, R\right)$ is the attenuation as defined by the expression (1) and $B$ is the system bandwidth around the center frequency $f_{c}$. The throughput, $T$, is defined as

$$
T=\frac{n \cdot t_{d}}{t_{t o t}}
$$

where $n$ refers to the number of correctly received packets, $t_{d}$ is the data packet length and $t_{t o t}$ is the total time needed on average to successfully transmit the $n$ packets. Averaging is performed over a number of different random deployments.

The total offered load, $L$, is determined by the packet arrival rate, $\lambda_{g}$ [packets/second], and the number of information generating nodes, $N$, as

$$
L=N \cdot \lambda_{g} \cdot t_{d}
$$

Packet arrivals are modeled as a Poisson process.

Simulation was conducted for an underwater network consisting of 20 nodes, 16 of which are relays, randomly placed over an area of $25 \mathrm{~km}^{2}$, three are information-generating mobile nodes, and one is a common sink. The total network area is divided into 16 squares and a relay is placed randomly within a square according to the uniform distribution. Hence, the density of relays is 0.64 nodes per square kilometer.

In the simulation, the data packets are generated by the mobile node and all are destined to the sink, while the fixed

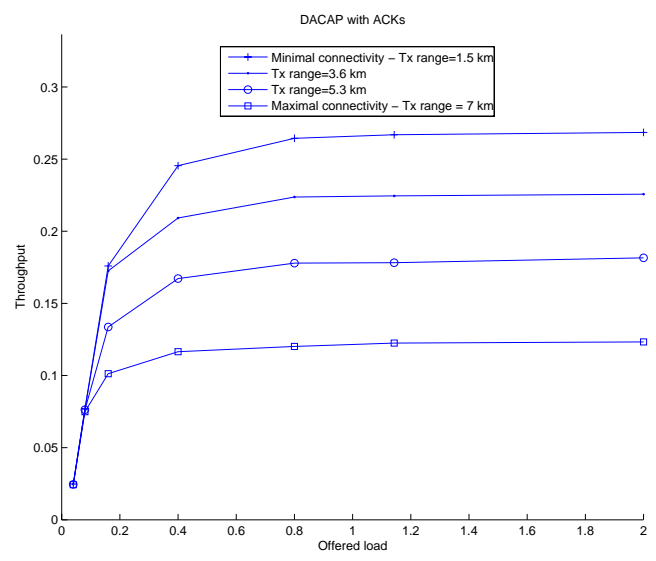

Fig. 3. Total throughput using the DACAP protocol with acknowledgments for different transmission ranges. Simulation time is 1000 seconds. 
nodes act as relays. On the down-link, the sink sends data packets to the mobile nodes through the network of relays. Hence, the total number of information generating nodes is $N=4$.

It is assumed that all the information generating nodes have the same rate $\lambda_{g}$. The center frequency used to evaluate the attenuation and the noise power is $f_{c}=35 \mathrm{kHz}$, and the bandwidth is $B=1 k H z$. The duration of the data packet is $t_{d}=2$ seconds. The total simulation time was set to 1000 seconds of network time.

Fig.2. and Fig.3. illustrate the results of the simulation. Fig. 2 shows the total energy consumed by all the nodes in the network during the simulation. The point labeled 'maximal connectivity' corresponds to the transmission range of $7 \mathrm{~km}$, which is sufficient to provide one-hop connectivity between maximally separated nodes within the $5 \times 5 \mathrm{~km}$ area. No routing is needed in this case, but the energy consumption is high. As the transmission range decreases, so does the energy consumption. Multi-hoping becomes necessary, which we accomplish using shortest path routing between the nodes. Since the relays are fixed, multi-hoping is easily implemented using pre-computed routing tables. The point labeled 'minimal connectivity' corresponds to the minimal transmission range for which connectivity is still guaranteed. Energy consumption is minimal at this transmission range. Hence, the optimal transmission range that minimizes the overall energy consumption is the range that corresponds to minimal connectivity. Reducing the transmission range beyond this point results in the loss of connectivity for some nodes. Simulation results demonstrate that substantial savings (about $65 \mathrm{~dB}$ ) in total power are available in the power-controlled scenario as compared to the maximally connected one.

Fig.3. shows the total throughput as a function of the total offered load, achieved with different transmission ranges. Each curve corresponds to a different transmission range. We observe that as the transmission range decreases from the $7 \mathrm{~km}$ to the $5.3 \mathrm{~km}, 3.6 \mathrm{~km}$ and $1.5 \mathrm{~km}$, the throughput performance improves. The throughput is maximized in the case of the minimal transmission range. Hence, the optimal transmission range-one that corresponds to minimal connectivity-simultaneoulsy results in maximal throughput and minimal energy consumption. This interesting observation further motivates the use of power control in underwater acoustic networks.

\section{RELATIONSHIP BETWEEN OPTIMAL TRANSMISSION RANGE AND NODE DENSITY}

To determine the optimal transmission range, $R_{o p t}$, one can start with the maximal transmission range and iteratively reduce it until minimal connectivity is reached. In the scenario presented in Fig.1., $R_{o p t}$ is $1.5 \mathrm{~km}$. The optimal power can be obtained using this value of $R_{o p t}$ in the expression (2).

The optimal transmission range can also be estimated from the node positions. Fig.4. illustrates this procedure for a network with six nodes and maximal inter-node distance of $7.5 \mathrm{~km}$. Shown in the figure are the possible connections between the nodes corresponding to four different transmission

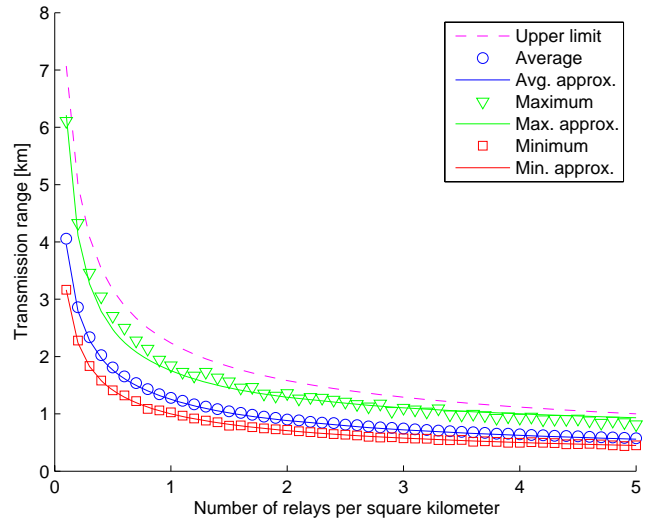

Fig. 5. Optimal transmission range at which minimal connectivity is achieved for different relay densities.

power levels. In a maximally connected network (a), a node can communicate with any other node in a single hop. In the scenario (b) some paths need multi-hoping. In a minimally connected scenario (c), the optimal transmission range $R_{o p t}$ can be calculated as the longest distance of the routing graph. If transmission power is further reduced, connectivity can not be guaranteed for all nodes, as illustrated in the scenario (d).

In practice, the optimal power can be computed centrally or in a distributed fashion. For a given density, a grid size is defined and a node is uniformly located within its grid. In this case, the optimal transmission range can be determined as a function of the relay density.

Fig.5. shows the average optimal transmission range as a function of the relay density (circles) for the network topology as that of Fig.1. Averaging is performed over 1000 random deployments. Shown also in the figure are the maximum (triangles) and the minimum (squares) of the optimal transmission range. The three values (average, maximum and minimum) clearly follow some trend, and this fact can be used to obtain closed form approximations. In particular, the average optimal

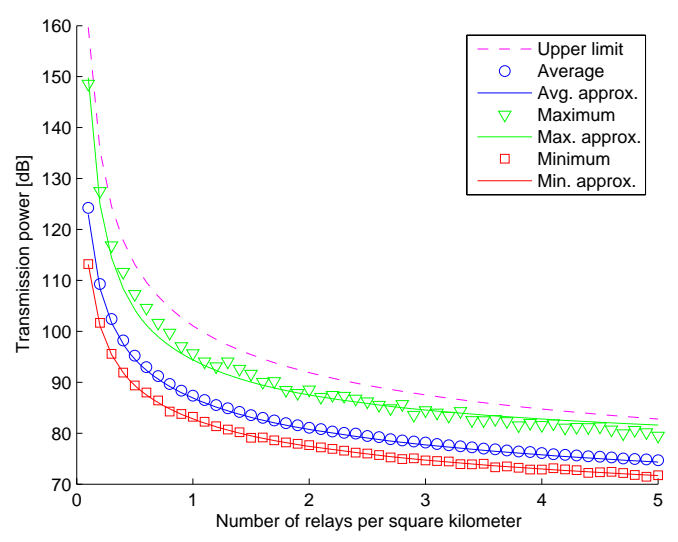

Fig. 6. Optimal transmission power at which minimal connectivity is achieved for different relay densities. 
a) Maximal connectivity - tx range $=7.5 \mathrm{~km}$

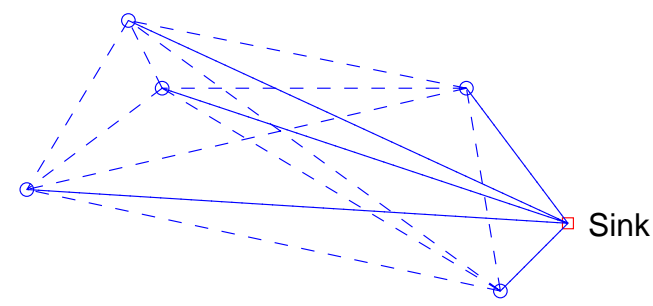

c) Minimal connectivity $-\mathrm{tx}$ range $=4.5 \mathrm{~km}$

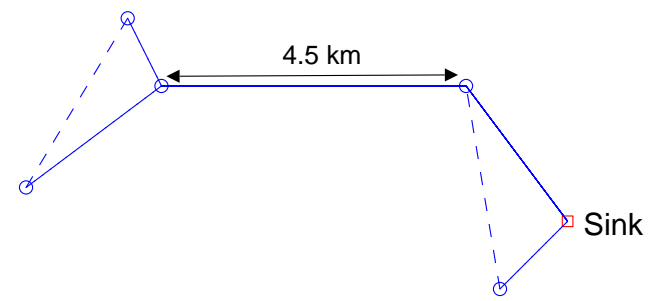

b) Medium connectivity - tx range $=6.5 \mathrm{~km}$

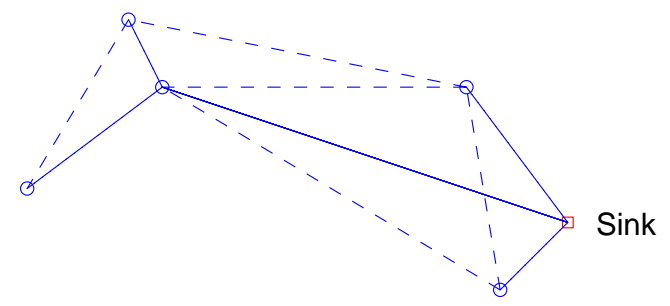

d) Loss of connectivity $-\mathrm{tx}$ range $=3.5 \mathrm{~km}$

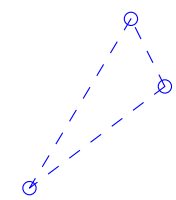

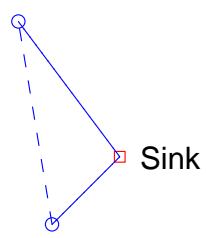

Fig. 4. Connectivity changes depending on the transmission power. Solid lines indicate shortest path routes from the sink (square on the right) to each of the nodes (circles). Dashed lines indicate other possible connections between relays.

transmission range can be approximated as

$$
\frac{1.25}{\sqrt{\rho}}
$$

where $\rho$ is the density given in the number of relays per square kilometer. In addition, the maximum and the minimum can be approximated as

$$
\frac{1.25}{\sqrt[3]{\rho^{2}}}+0.5
$$

and

$$
\frac{1}{\sqrt{\rho}}
$$

respectively. These approximations are shown in solid lines. The approximation for the minimal radius has an intuitive analytical explanation - it corresponds to placing each node in the center of its square. The approximation for the maximal radius, however, does not have a similar explanation. Maximal separation between two nodes is $\sqrt{\frac{5}{\rho}}$, which places an upper bound on the average maximal radius. This bound is shown in a dashed line in Fig.5.

Once the transmission range is defined, transmission power can be calculated from the expression (2). Fig.6. shows the transmission power corresponding to the average (circles), maximum (triangles) and minimum (squares) optimal transmission ranges, as well as the approximations obtained using the transmission range from the expressions (5), (6) and (7).
These approximations provide a simple tool that can be used to estimate the optimal transmission range in practical systems with a large number of nodes where measurement of inter-node distances may be difficult.

\section{CONCLUSION}

In this paper, power adjustment for DACAP has been discussed with the goal of making this MAC layer protocol applicable to an underwater acoustic network with arbitrary coverage. By changing the transmission power, energy consumption can be minimized and simultaneously throughput can be maximized. The optimal transmission power is found to be that power which corresponds to minimal connectivity. In a practical system, power control can be implemented in a distributed manner based on the knowledge or the measurement of inter-node distances. For a network with uniform node distribution the optimal transmission power can be determined as a function of the node density.

\section{ACKNOWLEDGMENT}

This work was supported by the following grants: NSF 0520075, NSF 0427502, NOAA /Sea Grant NA060AR4170019. 


\section{REFERENCES}

[1] B. Peleato and M. Stojanovic, "A MAC protocol for Ad Hoc Underwater Acoustic Sensor Networks," WUWNet'06, pp. 113-115, September 2006.

[2] M. Stojanovic, "On the Relantionship Between Capacity and Distance in a Underwater Acoustic Communication Channel," WUWNet'06, September 2006.

[3] N. Abramson, "Development of the ALOHAnet," IEEE Trans. Information Theory, pp. 119-123, 1985.

[4] P. Karn, "MACA - A new channel access method for a packet radio," ARRL/CRRL Amateur Radio 9th Computer Networking Conference, 1990.

[5] J. Rice, "Seaweb acoustic communication and navigation networks," in Proc. Conf. Underwater Acoustic Measurements: Technologies and Results, June 2005.

[6] M. Molins and M. Stojanovic, "Slotted FAMA: A MAC Protocol for Underwater Acoustic Networks," Proc. IEEE Oceans Conference 2006, 2006.

[7] X. Guo, M. Frater, and M. Ryan, "A Propagation-delay-tolerant Collision Avoidance Protocol for Underwater Acoustic Sensor Networks," Proc. IEEE Oceans Conference 2006, 2006.

[8] V. Rodoplu and M. Park, "An energy-efficient mac protocol for underwater wireless acoustic networks," in MTS/IEEE OCEANS, 2005.

[9] W. Ye and J. Heidemann, "Ultra-low duty cycle MAC with scheduled channel polling," Tech. Report ISI-TR-604, USC/ISI, 2005.

[10] J. Deng, Y. Han, P. Chen, and P. Varshney, "Optimum Transmission Range for Wireless Ad Hoc Networks," Wireless Communications and Networking Conference, WCNC 2004 IEEE, vol. 2, pp. 1024-1029, March 2004. 